\title{
It is time to move forward into the era of Theranostics
}

\author{
Hojjat Ahmadzadehfar ${ }^{*}$ and Markus Essler
}

\begin{abstract}
Radionuclide therapy, which until 15 years ago included only a few approved therapies, is gaining importance in the treatment of various malignancies. The future of oncology will not be limited to surgery, chemo-, antibody therapies or external radiation; it will include targeted therapy with radionuclides, which will become the standard of care for a variety of malignant diseases in combination or as an alternative to other therapies. Therefore there is a need to train Nuclear Oncologists, who are able to approach oncological diseases, promote development of radiopharmacy, understand the biology of radionuclide treatment, apply radionuclide treatments and be able to use molecular imaging such as PET/CT and SPECT/CT for treatment planning and dosimetry.
\end{abstract}

Keywords: Theranostics, Radionuclide therapy, Oncology, Fellowship

Abbreviations: $C T$, computed tomography; PET, positron emission tomography; PSMA, Prostate specific membrane antigen; SPECT, single-photon emission computed tomography

\section{Dear Editor,}

It is important to keep in mind that our specialty (Nuclear Medicine) is not confined to diagnostic imaging and, as a matter of fact, never was in the past. We are living in exciting times featuring revolutionary development of radionuclide therapy as well as tumour imaging.

Radionuclide therapy, which until 15 years ago included only a few approved therapies, is gaining importance in the treatment of various malignancies. The future of oncology will not be limited to surgery, chemo- and antibody therapies or external radiation; it will include targeted therapy with radionuclides, which will become the standard of care for a variety of malignant diseases in combination or as an alternative to other therapies.

Currently, in many Nuclear Medicine centres in addition to radioiodine therapy for thyroid cancer, radionuclide therapies such as radioembolisation of liver tumours and radiopeptide- and radioimmunotherapies for neuroendocrine tumours and lymphomas, as well as treatment of painful bone metastases with different radionuclides, are being performed [1-5]. Most importantly, radioligand therapy for metastatic prostate cancer with Lu-177-PSMA, which has been established in some

\footnotetext{
* Correspondence: hojjat.ahmadzadehfar@ukbonn.de

Department of Nuclear Medicine, University Hospital Bonn, Sigmund-Freud-Str. 25, 53127 Bonn, Germany
}

centres, shows remarkable treatment response and prolongation of overall survival in patients who do not have any other treatment options [6-10]; this further demonstrates the increasing importance of our specialty. Treatment of metastatic melanoma with melanin-binding benzamides, radioimmunotherapy for pancreatic cancer, antibody therapy for colorectal cancer and radionuclide therapy for leukaemia will be routinely offered therapies in the near future in combination with other therapies [11-14]. The chemokine ligand CXCR4 has been labelled with $177-\mathrm{Lu}$ and successfully used in the treatment of multiple myeloma patients with widespread refractory disease [15].

The rapid development of radiochemistry and radiopharmacy along with breakthroughs in immunology and cell biology highlights the future importance of Nuclear Medicine. Parallel to the advancement of radionuclide therapies, we have been experiencing revolutionary growth in diagnostic imaging agents with new tracers for brain imaging as well as tumour imaging. These advances have made our specialty grow into a highly complex and unique field clearly separated from diagnostic radiology and radiation oncology.

It is time to move forward into a new era, a time of theranostics. It is time to move forward to plan and develop a new subspecialty for Nuclear Medicine 
physicians called "Nuclear Oncology" within the next 10 years. These specialists should be able to deal with cancer patients, approach oncological diseases, promote the development of radiopharmacy, understand the biology of radionuclide treatment, apply radionuclide treatments and be able to use molecular imaging such as $\mathrm{PET} / \mathrm{CT}$ and SPECT/CT for treatment planning and dosimetry. It is mandatory for such specialists to be familiar with different oncologic therapies such as chemotherapy or immune therapy for better collaboration with all other specialties dealing with the treatment of cancer patients, including surgery, oncology, urology, gynaecology, paediatrics and pathology.

We propose the establishment of a curriculum for the training of nuclear medicine specialists to become a certified nuclear oncologist. The training should include a program of 1 year with rotations into specialised therapy units, PET/CT-imaging and radiopharmacy as well as outpatient care in oncology, radiation oncology and palliative medicine. During this entire period, collaboration with other specialties in oncology and participation in interdisciplinary tumour boards at a comprehensive cancer centre will be required. Apart from Nuclear Medicine learning resources, specific chapters of a medical oncology textbook, such as Cancer by DeVita et al. [16], should be defined as learning books for the final board certification exam taken by the candidates. In our opinion, Nuclear Oncologists will be an elite force to further develop our specialty and to improve patient care at highly specialised centres.

\section{Acknowledgements}

Not applicable

\section{Funding}

No funding

Availability of data and materials

Data sharing is not applicable to this article as no datasets were generated or analysed during the current study.

\section{Authors' contributions}

HA and ME wrote the letter together. HA and ME planed together the curriculum of the above-mentioned fellowship program. Both authors read and approved the final manuscript.

\section{Ethics approval and consent to participate}

Not applicable

\section{Consent for publication}

Not applicable

\section{Competing interests}

Both authors declare that they have no competing interests.

\section{Publisher's Note}

Springer Nature remains neutral with regard to jurisdictional claims in published maps and institutional affiliations.
Received: 21 December 2017 Accepted: 22 January 2018

Published online: 30 January 2018

References

1. Braat AJ, Smits ML, Braat MN, van den Hoven AF, Prince JF, de Jong HW, et al. (9)(0)Y hepatic radioembolization: an update on current practice and recent developments. J Nucl Med. 2015;56:1079-87. https://doi.org/10.2967/ jnumed.115.157446.

2. Yordanova A, Mayer K, Brossart P, Gonzalez-Carmona MA, Strassburg CP, Essler M, et al. Safety of multiple repeated cycles of 177Lu-octreotate in patients with recurrent neuroendocrine tumour. Eur J Nucl Med Mol Imaging. 2017;44:1207-14. https://doi.org/10.1007/s00259-017-3652-1.

3. Bodei L, Cremonesi M, Grana CM, Chinol M, Baio SM, Severi S, et al. Yttriumlabelled peptides for therapy of NET. Eur J Nucl Med Mol Imaging. 2012; 39(Suppl 1):S93-102. https://doi.org/10.1007/s00259-011-2002-y.

4. Tennvall J, Fischer M, Bischof Delaloye A, Bombardieri E, Bodei L, Giammarile $F$, et al. EANM procedure guideline for radioimmunotherapy for B-cell lymphoma with 90Y-radiolabelled ibritumomab tiuxetan (Zevalin). Eur J Nucl Med Mol Imaging. 2007;34: 616-22. https://doi.org/10.1007/s00259-007-0372-y.

5. Liepe K, Kotzerke J. Internal radiotherapy of painful bone metastases. Methods. 2011:55:258-70. https://doi.org/10.1016/j.ymeth.2011.07.001.

6. Ahmadzadehfar H, Rahbar K, Kurpig S, Bogemann M, Claesener M, Eppard $\mathrm{E}$, et al. Early side effects and first results of radioligand therapy with (177)Lu-DKFZ-617 PSMA of castrate-resistant metastatic prostate cancer: a two-centre study. EJNMMI Res. 2015;5:114. https://doi.org/10. 1186/s13550-015-0114-2.

7. Ahmadzadehfar H, Wegen S, Yordanova A, Fimmers R, Kurpig S, Eppard E, et al. Overall survival and response pattern of castration-resistant metastatic prostate cancer to multiple cycles of radioligand therapy using [177Lu]LuPSMA-617. Eur J Nucl Med Mol Imaging. 2017;44:1448-54. https://doi.org/10. 1007/s00259-017-3716-2.

8. Ahmadzadehfar H, Zimbelmann S, Yordanova A, Fimmers R, Kurpig S, Eppard E, et al. Radioligand therapy of metastatic prostate cancer using 177Lu-PSMA-617 after radiation exposure to 223Ra-dichloride. Oncotarget. 2017:8:55567-74. https://doi.org/10.18632/oncotarget.15698.

9. Yordanova A, Becker A, Eppard E, Kurpig S, Fisang C, Feldmann G, et al. The impact of repeated cycles of radioligand therapy using [177Lu]Lu-PSMA-617 on renal function in patients with hormone refractory metastatic prostate cancer. Eur J Nucl Med Mol Imaging. 2017;44(9):1473. https://doi.org/10. 1007/s00259-017-3681-9.

10. Rahbar K, Ahmadzadehfar H, Kratochwil C, Haberkorn U, Schafers M, Essler $M$, et al. German multicenter study investigating 177Lu-PSMA-617 radioligand therapy in advanced prostate cancer patients. J Nucl Med. 2017 58:85-90. https://doi.org/10.2967/jnumed.116.183194.

11. Mier W, Kratochwil C, Hassel JC, Giesel FL, Beijer B, Babich JW, et al. Radiopharmaceutical therapy of patients with metastasized melanoma with the melanin-binding benzamide 131I-BA52. J Nucl Med. 2014;55:9-14. https://doi.org/10.2967/jnumed.112.112789.

12. Sultana A, Shore S, Raraty MG, Vinjamuri S, Evans JE, Smith CT, et al. Randomised phase $|/| I$ trial assessing the safety and efficacy of radiolabelled anti-carcinoembryonic antigen I(131) KAb201 antibodies given intra-arterially or intravenously in patients with unresectable pancreatic adenocarcinoma. BMC Cancer. 2009;9:66. https://doi.org/10. $1186 / 1471-2407-9-66$

13. Sahlmann CO, Homayounfar K, Niessner M, Dyczkowski J, Conradi LC, Braulke $F$, et al. Repeated adjuvant anti-CEA radioimmunotherapy after resection of colorectal liver metastases: safety, feasibility, and long-term efficacy results of a prospective phase 2 study. Cancer. 2017;123:638-49. https://doi.org/10.1002/cncr.30390.

14. Hagemann UB, Wickstroem K, Wang E, Shea AO, Sponheim K, Karlsson J et al. In vitro and in vivo efficacy of a novel CD33-targeted thorium-227 conjugate for the treatment of acute myeloid Leukemia. Mol Cancer Ther. 2016;15:2422-31. https://doi.org/10.1158/1535-7163.MCT-16-0251.

15. Lapa C, Herrmann K, Schirbel A, Hanscheid H, Luckerath K, Schottelius M, et al. CXCR4-directed endoradiotherapy induces high response rates in extramedullary relapsed multiple myeloma. Theranostics. 2017;7:1589-97. https://doi.org/10.7150/thno.19050.

16. DeVita VT, Lawrence TS, Rosenberg SA. Devita, Hellman, and Rosenberg's cancer : principles \& practice of oncology. 10th ed. Philadelphia: Wolters Kluwer; 2015. 